\title{
L'atelier du traducteur. Benjamin et les Tableaux parisiens
}

Gérard Raulet

\section{OpenEdition}

Journals

Édition électronique

URL : http://journals.openedition.org/rief/656

DOI : 10.4000/rief.656

ISSN : 2240-7456

Éditeur

Seminario di filologia francese

Référence électronique

Gérard Raulet, "L'atelier du traducteur. Benjamin et les Tableaux parisiens », Revue italienne d'études françaises [En ligne], 4 | 2014, mis en ligne le 15 décembre 2014, consulté le 19 avril 2019. URL : http:// journals.openedition.org/rief/656 ; DOI : 10.4000/rief.656

Ce document a été généré automatiquement le 19 avril 2019

\section{(c) (i) (9)}

Les contenus de la RIEF sont mis à disposition selon les termes de la Licence Creative Commons Attribution - Pas d'Utilisation Commerciale - Pas de Modification 4.0 International. 


\title{
L'atelier du traducteur. Benjamin et les Tableaux parisiens
}

\author{
Gérard Raulet
}

Dès le début de mes études j'ai commencé à m'intéresser de très près à la littérature française. Il en résulta quelques traductions de Baudelaire, de

Proust, mais surtout une réflexion sur les problèmes linguistiques et philosophiques de la traduction que j'ai tenté de cerner dans un essai intitulé « Sur la tâche du traducteur ». ${ }^{1}$

1 En 1923 parut à Heidelberg dans une édition de qualité un mince volume bilingue intitulé Charles Baudelaire, Tableaux parisiens, traduction allemande avec une préface sur la tâche du traducteur, par Walter Benjamin'2. Si la préface compte parmi les textes les plus commentés de Benjamin, les traductions n'ont en revanche guère inspiré les interprètes. On tentera de suggérer ici que ces deux effets radicalement opposés sont en réalité les deux faces d'une même médaille.

2 Le recueil de 1923 contient dix-sept des dix-huit poèmes des Tableaux parisiens. Benjamin semble s'être cassé les dents sur À une mendiante rousse, qu'il a remplacé par La lune offensée - qui ne parut en fait que dans la troisième édition des Fleurs du mal en mai $1868^{3}$. Tout autant que le choix des autres poèmes des Fleurs du mal, sur lequel il serait bon que les interprètes s'interrogent, ce choix par défaut mérite l'attention; c'est pourquoi nous en proposons en exemple les différentes versions.

3 Aux dix-huit traductions publiées en 1923 s'ajoutent quatre poèmes publiés dans la revue Vers und Prosa des éditions Ernst Rowohlt en $1924^{4}$. Signe tangible que Benjamin ne considérait pas sa tâche de traducteur comme achevée. En tout ou en partie, il traduisit trente-cinq poèmes de Baudelaire dont on retrouve la trace dans neuf documents dactylographiés ou manuscrits ${ }^{5}$. La liasse manuscrite la plus ancienne remonte selon Scholem à $1915^{6}$; elle comprend vingt-sept poèmes, dont certains ne sont que partiellement traduits ${ }^{7}$. Une deuxième liasse, moins volumineuse, date, toujours selon 
Scholem, des années 1916 et $1917^{8}$. Un troisième manuscrit (Ms 151r), postérieur à 1917, contient une traduction de la deuxième strophe du poème Recueillement ${ }^{9}$ intitulée Vorbereitung. Alors même que le recueil publié chez Weissbach n'était pas encore paru, Benjamin pensait déjà à une édition plus importante. En janvier 1921, il écrit à Weissbach :

Je continue de travailler à une traduction des Fleurs du mal, non pas dans l'intention de transposer l'ensemble de l'ouvrage mais d'en proposer un choix couvrant la plus grande partie des cycles, sinon tous. Il est prévisible que ce travail prendra encore beaucoup de temps et ne pourra guère être achevé avant un délai de cinq ans. Il va de soi que je vous soumettrai en premier également ce livre. Mais pour le cas où nous ne pourrions parvenir à un contrat je souhaiterais me réserver le droit de reprendre aussi dans ce volume les poèmes des Tableaux parisiens parus chez vous. ${ }^{10}$

4 À ses yeux, le recueil publié n'était qu'un premier essai, auquel toutefois il attachait manifestement une grande importance, ne cessant de le remettre sur le métier. En mars il demande à Weissbach de lui renvoyer son manuscrit afin, dit-il, d'y apporter des corrections substantielles et d'y ajouter la deuxième partie du Cygne, entre-temps achevée ${ }^{11}$. Pour l'interprète cette traduction est l'une des plus instructives car, commencée dès 1915, Benjamin ne cessa de la retravailler entre 1920 et 1923. Nous donnons en annexe, à titre d'exemple, la première strophe.

5 Ce n'est que le 30 juin 1922 qu'il envoie le nouveau manuscrit enfin complété, mais dans lequel il manque toujours À une mendiante rousse ${ }^{12}$, remplacé par La lune offensée. Quant aux différents essais de traduction qui ne furent pas intégrés dans cette édition, les Gesammelte Schriften donnent à tort l'impression que les traductions rassemblées sous le titre Übertragungen aus anderen Teilen der 'Fleurs du mal' [Traductions d'autres parties des Fleurs du mal] sont de quelque façon une version finalisée, sinon autorisée. Or, elles ne parurent sous aucune forme. Il ne s'agit que de traductions plus ou moins abouties qu'il faut donc réinscrire dans le travail de longue haleine dont les différentes liasses donnent une image. En effet, quoique le recueil de 1923 n'ait pas eu de suite (ni l'édition populaire un temps envisagée, ni le recueil plus important auquel rêvait Benjamin), de toute évidence les exercices de traduction - traductions nouvelles ou reprises - se poursuivirent bien au-delà.

6 La plaquette publiée chez l'éditeur Weissbach n'est, comme souvent chez Benjamin, que la partie émergée d'un massif dont les plaques tectoniques s'étendent plus largement et plus profondément dans son œuvre. Si on en suit les traces, elles prennent des proportions démesurées. Il ne s'agissait pas uniquement de traduire quelques poèmes de Baudelaire, il s'agissait en même temps - et ce n'est qu'un premier niveau - d'ébaucher une théorie de la traduction à propos de laquelle on peut imaginer qu'elle devrait aussi s'appliquer aux traductions de Proust. Il s'agissait aussi, toujours en même temps, de percer à jour le monde de Baudelaire et d'alimenter ainsi le grand projet sur la modernité. Les différents textes qui peuvent être associés aux traductions, même stricto sensu, montrent non seulement que l'épisode de la traduction des Tableaux parisiens s'est poursuivi dans le temps mais qu'il s'est accompagné de notes qui se fondent tout naturellement dans le projet d'un ouvrage sur Baudelaire et/ou sur les passages parisiens. Dans l'exposé français des Passages parisiens, qui fut écrit en mars 1939, le troisième vers du Cygne II est placé en exergue de la quatrième partie. En 1939 aussi Benjamin rédige en français une Note sur les tableaux parisiens qui témoigne des liens étroits entre son travail sur les poèmes et sa réflexion sur Baudelaire comme poète de la modernité. Enfin, les archives parisiennes contiennent des transcriptions datant des derniers mois à Paris, 
notamment une version de La lune offensée qui porte encore le titre Die Kränkung der Mondgöttin, et non le titre plus allégorique Die Kränkung der Luna ${ }^{13}$. Enfin les liasses manuscrites intitulées «Notes sur quelques poèmes, des Fleurs du mal de Charles Baudelaire $~^{14}$, qui remontent à la même époque, ne peuvent certes pas être rapportées de façon univoque à l'entreprise de traduction ${ }^{15}$ mais plusieurs (notamment Ms 1774-1776 et Ms 1826) témoignent de la poursuite d'une réflexion approfondie sur les poèmes. Dans une lettre à Max Horkheimer du 24 juin 1939, Benjamin annonce que la nouvelle mouture du chapitre sur le flâneur de son Charles Baudelaire s'ouvrira par une analyse des Sept vieillards.

7 La présente note n'a aucune prétention philologique ni interprétative. Elle s'en garde même expressément car la tentation est grande de plaquer sur la pratique du traducteur les réflexions du théoricien. $\mathrm{Si}$, comme peuvent le donner à penser les exemples proposés ici, cette approche n'est pas complètement erronée, elle n'en néglige pas moins l'approche symétrique, qui correspond tout autant, sinon plus, à l'idée que Benjamin se faisait de son métier et de son statut d'écrivain : le traducteur comme le critique est un (re-)créateur qui, dans le cas extrême, sauve les œuvres en même temps qu'il achève leur destruction.

8 Cela étant dit, je ne pense pas non plus que Benjamin ait voulu ni détruire, ni sauver Baudelaire, c'est-à-dire qu'il ait de quelque façon forcé le trait pour démontrer sa thèse. L'œuvre de Baudelaire contenait bien assez de tension entre perdition et rédemption pour qu'il n'ait justement à développer que ce qui est selon lui - à tort ou à raison - le propre de ses traductions : la fidélité, au sens particulier où il l'entend. C'est, dans le court prière d'insérer qu'il rédige en réponse à une demande de Weissbach, la vertu dont il se réclame :

Votre souhait de recevoir une brève annonce m'a plongé dans la perplexité ; avec l'aide d'un ami j'ai finalement concocté ceci. Le cycle des Fleurs du mal publié ici contient de nombreux poèmes qui paraissent pour la première fois en traduction allemande. Ce qui distingue cette traduction est, d'une part, que l'impératif de fidélité exposé de façon irréfutable par le traducteur dans sa préface a été scrupuleusement mis en oeuvre et que, d'autre part, la matière poétique est rendue de façon convaincante. Tous les admirateurs du grand poète salueront le fait sans précédent que chaque traduction soit accompagnée du texte original dans une version philologiquement correcte. ${ }^{16}$

Il reste que la façon dont Benjamin met en œuvre la fidélité qu'il revendique prend des formes très expérimentales. Il n'est pas exclu qu'elles aient rebuté le lecteur, fût-il déjà aguerri par les pratiques de Stefan George. Le tirage modeste de 500 exemplaires fit long feu et l'édition populaire un temps envisagée ne vit jamais le jour. Il est également intéressant de relever que les poèmes publiés un an plus tard dans la revue Vers und Prosa présentent des divergences de ponctuation par rapport au tapuscrit (et aux corrections manuscrites qui y sont portées) - trace, sans doute, d'une intervention de l'éditeur. Benjamin ne se contente pas du renoncement aux majuscules, il expérimente une mise en forme typographique jouant sur l'espace entre les mots ${ }^{17}$. S'il ne s'agit des Calligrammes d'Apollinaire, qu'il devait aussi avoir en tête, il peut s'agir d'une tentative pour compenser le rendu toujours insuffisamment « fidèle » de la « matière poétique » par une composition à la fois rythmique et visuelle qui donne aussi à penser que Benjamin, 
comme les surréalistes, ne s'en tient plus à la séparation canonique entre les arts. Le 25 décembre 1920 déjà il fait part à Weissbach de son souhait de parler de la mise en page et de la typographie. Il ne cessera d'y revenir jusqu'à la parution. Le 4 avril 1921 il écrit à Weissbach qu'il entend user au minimum de la ponctuation et il demande ensuite à voir à quoi ressemble, une fois imprimé, un poème sans aucun signe de ponctuation. Finalement il se résout à en faire un usage parcimonieux ${ }^{18}$. Dans la traduction de la deuxième partie du Cygne il est manifeste que Benjamin a commencé par chercher à couler sa diction dans le moule de la forme baudelairienne ${ }^{19}$. A cet égard le problème de la métrique, sur lequel Benjamin s'appesantit dans des lettres à Florens Christian Rang et à Hofmannsthal, est l'arbre qui cache la forêt.

Il nous semble que c'est dans cette direction que devraient s'orienter les recherches sur les «traductions» de Benjamin - tant elles sont parfois contestables sur le plan de la « fidélité » à la lettre de l'original et mystérieuses quant à leurs propres techniques. Pour le reste, en effet, tout peut être dit assez rapidement et les travaux universitaires qui se sont frottés à ces traductions ont en général tourné court. On peut à coup sûr y voir une tendance à l'allégorie ; c'est indéniable. Elle est confirmée par la lettre à Hofmannsthal de janvier 1924 :

Je suis convaincu qu'au bout du compte seule la maîtrise de la métrique permet à une traduction des Fleurs du mal de rendre compte du style baudelairien avec plus d'intensité que ne le font les miennes; il s'agit d'un style qui m'a finalement plus fasciné que tout le reste et que j'appellerais volontiers un baroque de la banalité, au sens où Claudel l'a qualifié de mélange entre le style de Racine et celui d'un reporter des années quarante du siècle passé. Bref, je voudrais encore une fois me mettre en chasse et tenter d'aborder ces registres linguistiques où le mot à la mode rencontre l'abstraction allégorique (Spleen et idéal) et, en même temps, atteindre sur ce terrain à une pareille clarté du mètre.

L'exemple proposé ci-après confirme indéniablement cette intention "allégorique" et "baroque". 1923, l'année où il "achève » sa traduction de la deuxième partie du Cygne, coïncide avec le début du travail sur l'Origine du drame baroque. Au contraire des premières ébauches, la version finale du Cygne II est marquée par l'abstraction: l'abandon presque complet des adjectifs conduit à l'opposition abrupte de l'ancien et du nouveau (« Die neue Stadt die alte »); le remplacement du mot allemand «Leiden» par le mot étranger «Melancholie » a un effet qu'on peut qualifier de conceptuel : il ne s'agit nullement d'un quelconque retour à la traduction littérale du terme baudelairien de "mélancolie», le simple usage du mot étranger, renforcé par sa mise en attaque du deuxième vers, a valeur quasi théorique et raccourcit encore le lien avec l'allégorie. En même temps on ne peut que constater les faiblesses des tentatives de Benjamin. Toute la matière concrète de l'image de Paris disparaît et, surtout, Benjamin réintroduit un "wie» (comme), une comparaison explicite, là où Baudelaire intériorise sans médiation le poids du souvenir : «Et mes chers souvenirs sont plus lourds que des rocs.» On observe en gros la même chose dans les traductions de La lune offensée, que nous avons retenues parce qu'elles couvrent, comme Le Cygne II, toute la durée de gestation du recueil de 1923. La première version manuscrite remonte à 1915. Après avoir esquissé des procédés techniques expérimentaux Benjamin en revient finalement, à son corps défendant sans doute, à une traduction «philosophique » qui force le trait dans le sens du message faute de pouvoir recréer une forme.

12 Il est toutefois difficile de tirer de ce genre de constatations plus qu'une analyse comparée, "traductologique", des procédés de l'original et de sa traduction - une analyse 
qui conduit immanquablement à la conclusion que Benjamin, décidément, n'est pas un "bon traducteur". Je pourrais en ce point céder à la convention et prendre un faux virage en disant que tel n'était pas son propos. Suivrait alors ce qui va suivre ici aussi, à savoir un rappel des conceptions très particulières que Benjamin avait de la traduction. Il restera en tout état de cause à se demander si ses prétentions théoriques ont vraiment trouvé un quelconque aboutissement dans sa pratique de traducteur. Rien n'est moins sûr - au risque de heurter les tabous. Aussi laborieusement qu'elles aient tenté de la pratiquer, elles illustrent dans le meilleur des cas l'intention théorique de l'interprète. Il en convient lui-même dans une lettre du 10 janvier 1924 à Florens Christian Rang :

Maintenant que l'ouvrage est là je me rends compte qu'il est finalement plus essentiel pour un auteur d'apparaitre en public avec ses productions problématiques plutôt qu'avec celles qui sont réussies, dans la mesure où la délivrance qu'on attend de l'ouvrage imprimé est plus grande dans le premier cas que dans le second. Le caractère éminemment problématique de cette traduction ne fait pour moi aucun doute car il lui manque tout simplement le tour de main, la maîtrise dans le domaine de la métrique. [...] Cela n'était naturellement pas rattrapable, seul un nouveau commencement peut avoir un sens. J'espère que cela me sera un jour possible. ${ }^{20}$

13 Pour Benjamin, la mission du traducteur ne consiste pas à rapprocher l'original du destinataire et à partir pour ce faire des attentes de ce dernier. Il se distingue en cela radicalement de la démarche herméneutique, que Schleiermacher résume par l'alternative suivante: «Ou bien le traducteur laisse l'auteur en paix autant que faire se peut et il conduit le lecteur dans sa direction; ou bien il laisse le lecteur autant que possible en paix et il conduit l'auteur dans la direction de ce dernier. » Benjamin partage avec Schleiermacher la conviction que la paraphrase et le mimétisme sont des pis-aller et qu'il faut plutôt éviter de mélanger les univers de langue et de compréhension dès lors qu'ils résistent à se fondre. Dans un entretien avec le critique Benjamin Crémieux, en 1927, il prête à ce dernier des propos qui pourraient être les siens : « Et troisièmement je ne suis en rien un adepte de la fidélité philologique, archaïsante, en matière de traduction $»^{21}$. Il dénonce ainsi le second contresens qu'on commet volontiers sur sa conception de la "tâche du traducteur ", après celui qui porte sur sa conception de la réception : l'enjeu n'est ni la restauration de l'original, ni la satisfaction des attentes du récepteur. Comme Schleiermacher, Benjamin se refuse à combler hâtivement l'abîme entre les langues et les cultures. La communication « authentique » ne consiste pas dans la transmission (Mitteilung) d'un message ou dans la compréhension (Verständigung) à tout prix.

14 Le petit texte de 1926 «Traductions » («Übersetzungen »), moins fréquemment exploité, résume sans détour les prémisses de la théorie benjaminienne de la traduction : «Celui qui traduit travaille dans deux langues ${ }^{22}$. Il ne s'agit pas, comme le disait déjà « La tâche du traducteur ", de faire en sorte que pour le récepteur la traduction « se lise comme une œuvre originale de sa propre langue ", mais de créer des ponts qui - je cite toujours « La

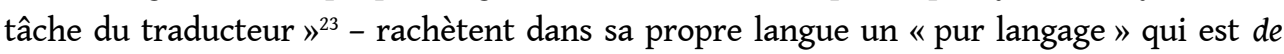
toute façon exilé dans une langue étrangère. Ce « langage originaire » n'est pas plus dans la langue de départ que dans la langue d'arrivée. Le but «idéal» (pour paraphraser Baudelaire, qui n'y croyait plus vraiment) serait de le « libérer en le transposant ».

15 «À partir des deux langues le traducteur construit quelque chose », dit le texte de 1926. On comprend mieux, à la lumière de cette déclaration, le sens de la «littéralité ». Une traduction, poursuit Benjamin, se construit « en mettant un vers sur l'autre, d'étage en 
étage, jusqu'à ce que, bien souvent, une erreur minime, au final, fasse s'écrouler l'ensemble, laissant le chant sans voix ". La vertu et le risque d'une telle technique est qu'elle ne sacrifie pas le matériau linguistique et poétique au « sens ».

C'est bien, en effet, ce qui se passe dans ses traductions, comme les spécimens présentés ici le démontrent. Et ce qu'ils démontrent aussi, par leur tentative désespérée pour compenser l'impossibilité de rendre le rythme par la métrique, c'est une tendance au mimétisme que par ailleurs il dénonce expressément. Il y a là un vrai problème philosophique qui va bien au-delà de la question de la traduction et qui touche au rôle fondamental du mimétisme dans la pensée de Benjamin, et en particulier dans sa pensée politique. Car, au fond, la seule alternative à l'impossibilité de la « communication » et de la " compréhension ", c'est le mimétisme. En cela que réside le lien non seulement entre l'essai sur le langage et celui sur la tâche du traducteur, mais également entre eux et les positions politiques des années trente. Dans les deux textes de 1933 sur la théorie de la ressemblance et le pouvoir mimétique ${ }^{24}$, au premier abord très abscons, il y va de la même chose que dans les textes sur la traduction ou sur la langue originelle - avec des perspectives civilisationnelles et politiques plus urgentes, compte tenu de la date. Ils sont écrits en réaction aux thèses de Caillois, du Collège de sociologie et du groupe Acéphale, et posent la question de la stratégie à adopter face à l'ennemi fasciste: la "barbarie positive» doit-elle aller jusqu'au comportement de la mante religieuse? Cette problématique est loin de ne concerner que le seul Benjamin. Adorno participa activement au débat et la parenté est indéniable entre la conception de la mimésis chez Caillois et ce qu'Adorno appellera la mimésis du mort (Mimesis ans Tote) - une expression qui vient d'ailleurs plus que vraisemblablement de Benjamin ; l'occurrence première que je connais est celle-ci : « Hugo ignore le pouvoir de figer les choses qui - s'il est pertinent de recourir à un concept biologique - se manifeste comme une sorte de mimésis de la mort dans la poésie de Baudelaire $»^{25}$.

Celle-ci serait la position de repli «matérialiste» d'une philosophie qui a perdu tout espoir de se situer en dehors de la réification. Il en va de même du Nom - comme chez Benjamin. Dans la Dialektik der Aufklärung le nom propre est utilisé comme un coup d'arrêt au jugement d'identité qui opère au moyen de la copule, de la même façon que la mimésis met hors circuit toute équivalence de choses non équivalentes. L'un et l'autre jouent l'immédiateté contre la médiation; ils prennent le parti de la "primauté de l'objet » qu'affirmera la Dialectique négative. Il s'agit de contrecarrer la logique de l'échange. 


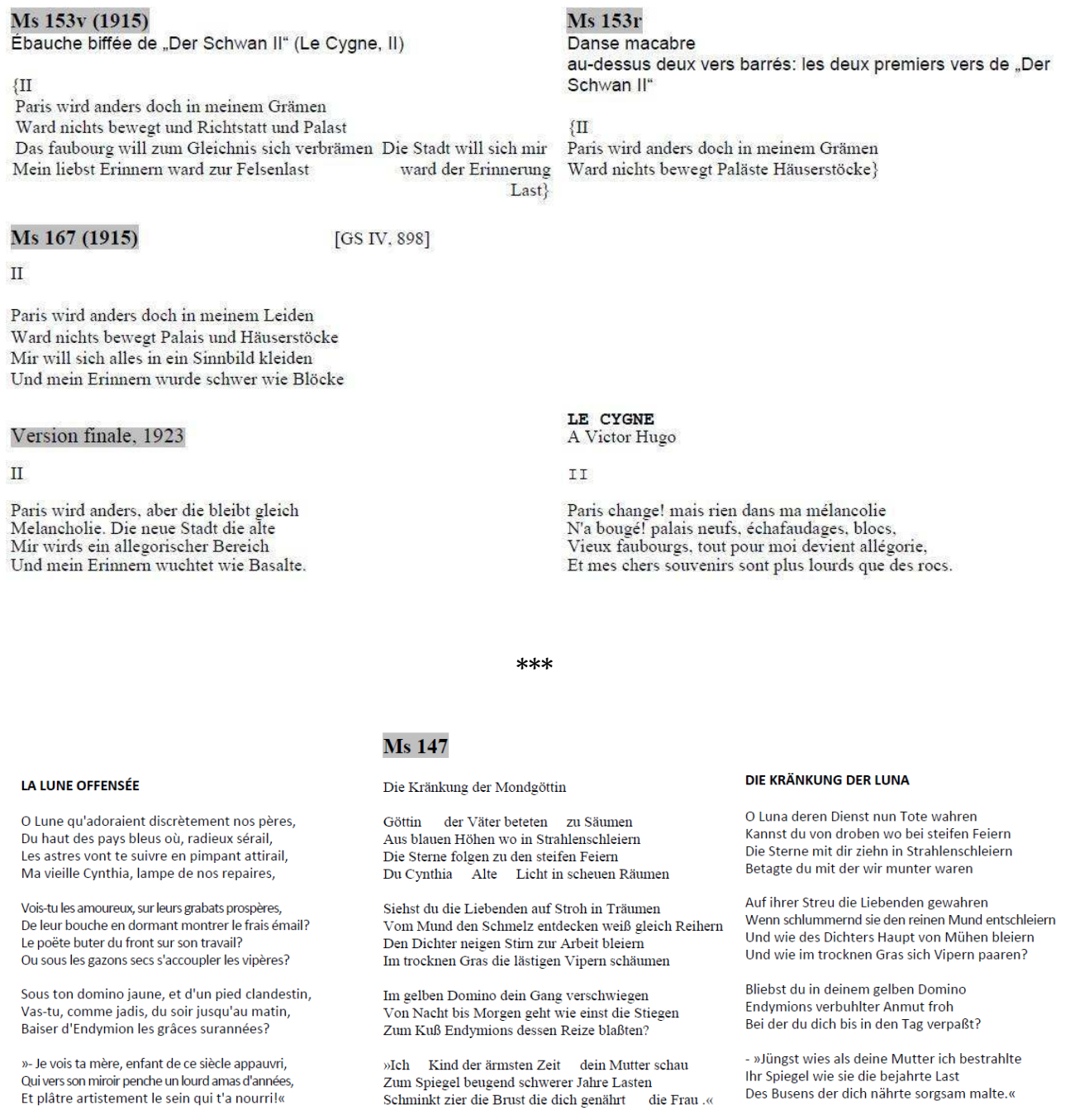

\section{NOTES}

1. W. Benjamin, Second curriculum vitae, in Gesammelte Schriften [désormais en abrégé: GS], Frankfurt/M., Suhrkamp, 1978, VI, p. 216 sq.

2. Charles Baudelaire, Tableaux parisiens. Deutsche Übertragung mit einem Vorwort über die Aufgabe des Übersetzers von Walter Benjamin, Heidelberg, Verlag von Richard Weißbach, « Die Drucke des Argonautenkreises ", 5, 1923.

3. Il fut publié une première fois en 1862 dans la revue L'Artiste, puis repris par Théophile Gautier dans la troisième édition (posthume) des Fleurs du mal.

4. «Baudelaire-Übertragungen", in Vers und Prosa, 1924, pp. 269-272 (cahier 8) : An den Leser, Frohsinn des Toten, Die Wanduhr, Einer Madonna. La liasse dont sont tirées ces versions contenait en fait sept poèmes. 
5. Dont il n'est pas possible de détailler ici la liste ; on se reportera aux Gesammelte Schriften, dans l'attente de l'édition nouvelle Werke und Nachlaß. Kritische Gesamtausgabe, t. VI: Charles Baudelaire. Tableaux parisiens.

6. Dans une lettre à Hofmannsthal de janvier 1924, Benjamin dit lui-même que neuf ans ont passé depuis ses premiers essais de traduction (Gesammelte Briefe, éd. par Christoph Gödde et Henri Lonitz, 6 tomes, Frankfurt/M., Suhrkamp, 1995 sq. - tome 2 : Briefe 1919-1924, Frankfurt/M., Suhrkamp, 1996, p. 410).

7. Mss 141, 146-150, 152-183: Der Untergang der Sonne, Die Kränkung der Mondgöttin, Trauriges

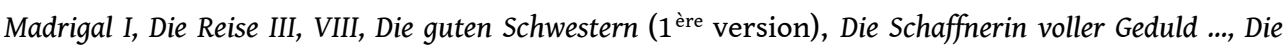
sieben Greise, Die Monddämmerung, Die Abenddämmerung, Die kleinen Alten IV, Der Schwan, Landschaft, Unterhaltung, Geistige Morgenröte, Totenreue, Die Riesin, Die kranke Muse, Vergessen hab ich nicht ..., Der Geist, Nebel und Regen, Die Katzen, Sisina, Herbstlied I, Der Tod der Liebenden, Die barmherzigen Schwestern ( $2^{\mathrm{e}}$ version), Die Zerstörung, Der Wein der Einsamen.

8. Mss 142-145 (Die Katzen, Vergessen hab ich nicht ..., Der Tod der Liebenden, Unterhaltung, Der Geist, Geistige Morgenröte, Herbstlied I, Die kranke Muse, Totenreue).

9. «Pendant que des mortels la multitude vile, / Sous le fouet du Plaisir, ce bourreau sans merci, / Va cueillir des remords dans la fête servile, / Ma Douleur, donne-moi la main ; viens par ici [... ]».

10. À R. Weißbach, 22.1.1921.

11. «Ich möchte in diesem eine Anzahl von Verbesserungen, die ich inzwischen vorgenommen habe anbringen, zugleich diejenigen Gedichte bezeichnen, die ich Ihnen für den Vorabdruck in den »Argonauten« vorschlage und endlich das eine noch fehlende Gedicht [Der Schwan II] einfügen ».

12. De ce poème il n'existe qu'un seul essai de traduction dans un manuscrit retrouvé à Paris à la Bibliothèque nationale.

13. Il s'agit du manuscrit 085v. Il est plausible que Benjamin avait toujours en vue une édition augmentée de ses traductions des Fleurs du mal et qu'une mise au propre des versions existantes ait été entreprise à cette fin.

14. « Notizen / Exzerpte zu einigen Gedichten aus : Charles Baudelaire, Fleurs du mal » (= Ms 1761-1815 \& 1826).

15. C'est notamment le cas des "Neuf thèses" sur le flâneur et sur l'empathie avec la marchandise (Ms 1816-1819, GS I-3, 1173-75), ou encore des notes Ms 1820-1825 (GS I-3, 1150-52) et Ms 1827-1833, qui se rattachent plus nettement au projet des Passages parisiens et du Charles Baudelaire.

16. À Richard Weissbach, le 12 octobre 1923.

17. Voir en annexe la première traduction de La lune offensée; nous avons tenté de reproduire les espaces. Dans la première ébauche du Cygne (voir également en annexe) l'intérêt pour le rythme l'emporte à tel point que Benjamin ne se préoccupe guère des contresens; ainsi il confond «échafaudage» avec «échafaud» (Richtstatt). La littéralité revendiquée dans l'essai "Sur la tâche du traducteur " n'a rien à voir avec la précision sémantique ; cela restera vrai dans les ébauches suivantes.

18. Lettre du 3 mai 1922 à Weissbach.

19. Voir l'absence de ponctuation dans la première version du Cygne II que nous proposons en annexe.

20. Gesammelte Briefe, op. cit., tome 2: Briefe 1919-1924, p. 407.

21. GS IV-1,2, p. 497.

22. «Wer übersetzt, arbeitet in zwei Sprachen. » (« Übersetzungen », GS III, p. 40).

23. GS IV-1, p. 18 sq.

24. « Lehre vom Ähnlichen » (1933) et « Über das mimetische Verfahren » (1934), GS II-1, 204-210 et $210-213$.

Revue italienne d'études françaises, 4 | 2014 
25. « Hugo ist das Erstarrungsvermögen fremd, das - wenn ein biologischer Begriff statthaft ist als eine Art Mimesis des Todes sich hundertfach in Baudelaires Dichtung kundtut.» (Das Paris des Second Empire, « Die Moderne », GS I, 587).

INDEX

Mots-clés : Baudelaire (Charles), traduction en allemand, Benjamin (Walter), Tableaux Parisiens 